\title{
In vivo hyperspectral imaging of skin malignant and benign tumors in visible spectrum
}

\author{
Ivan A. Bratchenko ${ }^{1 *}$, Violetta P. Sherendak ${ }^{1}$, Oleg O. Myakinin ${ }^{1}$, Dmitry N. Artemyev ${ }^{1}$, \\ Alexander A. Moryatov ${ }^{2,3}$, Ekaterina Borisova ${ }^{4}$, Latchezar Avramov ${ }^{4}$, Larisa A. Zherdeva ${ }^{1}$, \\ Andrey E. Orlov ${ }^{3}$, Sergey V. Kozlov ${ }^{2,3}$, and Valery P. Zakharov ${ }^{1}$ \\ ${ }^{1}$ Department of Laser and Biotechnical Systems, Samara National Research University, 34 Moskovskoe Shosse, Samara \\ 443086, Russian Federation \\ ${ }^{2}$ Department of Oncology, Samara State Medical University, 89 Chapayevskaya St., Samara 443099, \\ Russian Federation \\ ${ }^{3}$ Samara Regional Clinical Oncology Dispensary, 50 Solnechnaya st., Samara 443031, Russian Federation \\ ${ }^{4}$ Institute of Electronics, Bulgarian Academy of Sciences, 72 Tzarigradsko chaussee blvd, Sofia 1784, Bulgaria \\ *e-mail: iabratchenko@gmail.com
}

\begin{abstract}
The paper presents analysis of hyperspectral images for human skin cancer pathologies diagnostics. Hyperspectral images data contained backscattered spectra of normal skin and tumors. Analysis of hyperspectral images provided information about hemoglobin and melanin content for the differentiation of malignant and benign skin neoplasms based on tissues optical density calculation. It was shown that the accuracy of cancerous tissues classification reaches $88 \%$ for proposed algorithm of hyperspectral images analysis. The proposed approach may be a rapid and reliable tool for skin oncological diseases screening. (C) 2018 Journal of Biomedical Photonics \& Engineering.
\end{abstract}

Keywords: hyperspectral imaging; backscattering spectroscopy; oncodermatology; oncological diagnostics; melanoma; nevi; carcinoma; melanin; hemoglobin content.

Paper \#3252 received 10 Sep 2017; revised manuscript received 2 Oct 2017; accepted for publication 4 Oct 2017; published online 16 Jan 2018. doi: 10.18287/JBPE17.04.010301.

\section{References}

1. All Cancers (excluding non-melanoma skin cancer). Estimated Incidence, Mortality and Prevalence Worldwide in 2012, GLOBOCAN.

2. D. V. Karpin, V. V. Starinskiy, G. V. Petrova (Eds.), The state of oncological care for the population in 2015 in Russia, MORI - P. Herzen Moscow Oncology Research Institute, Moscow (2016) [in Russian].

3. A. V. Ermakov, "Early diagnosis and prophylaxis for cutaneous melanoma," Oncosurgery 5(3), 52-58 (2013) [in Russian].

4. N. R. Abbasi, H. M. Shaw, D. S. Rigel, R. J. Friedman, W. H. McCarthy, I. Osman, A. W. Kopf, and D. Polsky, "Early diagnosis of cutaneous melanoma: revisiting the ABCD criteria," JAMA 292(22), 27712776 (2004).

5. F. M. Walter, A. T. Prevost, J. Vasconcelos, P. N. Hall, N. P. Burrows, H. C. Morris, A. L. Kinmonth, and J. D. Emery, "Using the 7-point checklist as a diagnostic aid for pigmented skin lesions in general practice: a diagnostic validation study," British Journal of General Practice 63(610), 345-353 (2013).

6. G. Argenziano, G. Fabbrocini, P. Carli, V. De Giorgi, E. Sammarco, and M. Delfino, "Epiluminescence microscopy for the diagnosis of doubtful melanocytic skin lesions," Archives of Dermatology 1341(12), 15631570 (1998).

7. C. Benvenuto-Andrade, S. W. Dusza, A. L. C. Agero, A. Scope, M. Rajadhyaksha, A. C. Halpern, and A. A. Marghoob, "Differences Between Polarized Light Dermoscopy and Immersion Contact Dermoscopy for the Evaluation of Skin Lesions," Archives of Dermatology 143(3), 329-338 (2007).

8. P. Bourne, C. Rosendahl, J. Keir, and A. Cameron, "BLINCK-A diagnostic algorithm for skin cancer diagnosis combining clinical features with dermatoscopy findings," Dermatology Practical \& Conceptual 2(2), 55-61 (2012). 
9. C. Rosendahl, G. Williams, D. Eley, T. Wilson, G. Canning, J. Keir, I. McColl, and D. Wilkinson, “The impact of subspecialization and dermatoscopy use on accuracy of melanoma diagnosis among primary care doctors in Australia," Journal of the American Academy of Dermatology 67(5), 846-852 (2012).

10. C. Massone, A. Di Stefani, and H. P. Soyer, "Dermoscopy for skin cancer detection," Current Opinion in Oncology 17, 147-153 (2005).

11. G. Lu, and B. Fei, "Medical hyperspectral imaging: a review," Journal of Biomedical Optics 19(1), 010901 (2014).

12. I. Diebele, I. Kuzmina, A. Lihachev, J. Kapostinsh, A. Derjabo, L. Valeine, and J. Spigulis, "Clinical evaluation of melanomas and common nevi by spectral imaging," Biomedical Optics Express 3(3), 467-472 (2012).

13. A. Machihin, and V. Pozhar, "Double-AOTF-based aberration-free spectral imaging endoscopic system for biomedical applications," Journal of Innovative Optical Health Sciences 8(3), 1541009 (2015).

14. I. A. Bratchenko, M. V. Alonova, O. O. Myakinin, A. A. Moryatov, S. V. Kozlov, and V. P. Zakharov, "Hyperspectral visualization of skin pathologies in visible region," Computer Optics 40(2), 240-248 (2016).

15. L. A. Zherdeva, I. A. Bratchenko, O. O. Myakinin, A. A. Moryatov, S. V. Kozlov, and V. P. Zakharov, "In vivo hyperspectral imaging and differentiation of skin cancer," Proceedings of SPIE 10024, 100244G (2016).

16. R. O. Duda, P. E. Hart, and D. G. Stork, Pattern Classification, 2nd ed., Wiley (2001).

17. A. N. Bashkatov, E. A. Genina, V. I. Kochubey, and V. V. Tuchin, "Optical properties of human skin, subcutaneous and mucous tissues in the wavelength range from 400 to $2000 \mathrm{~nm}$," Journal of Physics D: Applied Physics 38(15), 2543-2555 (2005).

18. R. L. van Veen, H. J. Sterenborg, A. Pifferi, A. Torricelli, E. Chikoidze, and R. Cubeddu, "Determination of visible near-IR absorption coefficients of mammalian fat using time- and spatially resolved diffuse reflectance and transmission spectroscopy," Journal of Biomedical Optics 10(5), 054004 (2005).

19. G. Zonios, J. Bykowski, and N. Kollias, "Skin melanin, hemoglobin, and light scattering properties can be quantitatively assessed in vivo using diffuse reflectance spectroscopy," Journal of Investigative Dermatology 117(6), 1452-1457 (2001).

20. V. P. Zakharov, I. A. Bratchenko, S. V. Kozlov, A. A. Moryatov, O. O. Myakinin, and D. N. Artemyev, "Twostep Raman spectroscopy method for tumor diagnosis," Proceedings of SPIE 9129, 91293V (2014).

21. I. A. Bratchenko, D. N. Artemyev, O. O. Myakinin, Y. A. Khristoforova, A. A. Moryatov, S.V. Kozlov, and V. P. Zakharov, "Combined Raman and autofluorescence ex vivo diagnostics of skin cancer in near-infrared and visible regions," Journal of Biomedical Optics 22(2), 027005 (2017).

22. E. G. Borisova, L. P. Angelova, and E. P. Pavlova, "Endogenous and Exogenous Fluorescence Skin Cancer Diagnostics for Clinical Applications," IEEE Journal of Selected Topics in Quantum Electronics 20(2), 211222 (2014).

23. J. Zhao, H. Lui, S. Kalia, and H. Zeng, "Real-time Raman spectroscopy for automatic in vivo skin cancer detection: an independent validation," Analytical and Bioanalytical Chemistry 407(27), 8373-8379 (2015).

24. S.-H. Tseng, P. Bargo, A. Durkin, and N. Kollias, "Chromophore concentrations, absorption and scattering properties of human skin in-vivo," Optics Express 17(17), 14599-14617 (2009).

25. D.T. Dicker, J. Lerner, P. Van Belle, S.F. Barth, D. Guerry 4th, M. Herlyn, D.E. Elder, and W.S. El-Deiry, "Differentiation of normal skin and melanoma using high resolution hyperspectral imaging," Cancer Biology \& Therapy 5(8), 1033-1038 (2006).

26. Z. Huang, H. Lui, D. I. McLean, M. Korbelik, and H. Zeng, "Raman Spectroscopy in Combination with Background Near-infrared Autofluorescence Enhances the In Vivo Assessment of Malignant Tissues," Photochemistry and Photobiology 81(5), 1219-1226 (2005).

27. W. Wang, J. Zhao, M. Short, and H. Zeng, "Real-time in vivo cancer diagnosis using raman spectroscopy," Journal of Biophotonics 8(7), 527-545 (2014).

28. X. Feng, A. J. Moy, H. T. M. Nguyen, J. Zhang, M. C. Fox, K. R. Sebastian, J. S. Reichenberg, M. K. Markey, and J. W. Tunnell, "Raman active components of skin cancer," Biomedical Optics Express 8(6), 2835-2850 (2017).

29. L. A. Austin, S. Osseiran, and C. L. Evans, "Raman technologies in cancer diagnostics," Analyst 141(2), 476503 (2016).

30. V. P. Zakharov, I. A. Bratchenko, D. N. Artemyev, O. O. Myakinin, D. V. Kornilin, S. V. Kozlov, and A. A. Moryatov, "Comparative analysis of combined spectral and optical tomography methods for detection of skin and lung cancers," Journal of Biomedical Optics 20(2), 025003 (2015).

\section{Introduction}

Malignant melanoma holds a special place among other skin malignancies. It is the most aggressive skin tumor and it is responsible for more than $85 \%$ of all lethal cases from malignant skin tumors. Skin melanoma is uneven with the highest incidence rates for Australia 
and New Zealand (up to 40 cases per 100.000 population), the United States of America (up to 21.1 cases per 100.000 population), and some countries in Europe [1]. In particular, in the United States in 2013, the disease was detected in 76,600 Americans, while $35 \%$ of sick people were under 45 years old. In Russia more than half a million new patients with malignant neoplasms are detected each year, and $14 \%$ of them are malignant skin tumors. Skin melanoma in Russia is less common (about 8500 cases every year - 3.97 new diseases per 100 thousand people), but also a high annual incidence rate from 4.55 to $6.1 \%$ per year can be stated for the last decade [2]. In Samara region a high incidence rates of malignant neoplasms is observed about 446.6 new cases per 100.000 population. One of the region features is a high incidence of skin tumors; about $18.6 \%$ of all new cancers are skin cancers, and Samara holds the first place in Russia according to this index in 2015.

Skin melanoma is characterized by a variety of clinical marks, and it is especially difficult to differentiate melanoma from other skin tumors in the initial stages of the disease. Potentially dangerous pigmented skin tumors can be detected by more than $90 \%$ of the population during visual examination [3]. Hyperdiagnosis of melanoma leads to an unreasonably high volume of surgical interventions and serious cosmetic defects. Despite the significant progress achieved in systemic drug therapy for skin melanoma, the results of 5-year survival are still most dependent on timely and effective diagnosis and adequate volume of surgical treatment. Therefore, design of methods for early skin cancers diagnostics remains in high demand.

Today common practice to increase accuracy of first-hand inspection is dermatoscopy analysis of skin neoplasms. Such an analysis provides the opportunity to enlarge the standard ABCD rule [4] or 7-point checklist [5] and involve additional criteria in suspicious skin tissues inspection. For example, dermatoscopy analysis may include monitoring of more specifically related tumor features like atypical pigmentation and vascular networks as well as so-called blue-whitish veil, which represents a gray-white pattern with well perceptible blue tone [6]. Unfortunately, the features' list has been originally borrowed from epiluminescence microscopy. In most cases, that means a special immersion interface should be used to get high-quality images in contrast with the ordinary $\mathrm{ABCD}$ rule or 7-point checklist, which, in general, do not requires any refractive equalization agents, but, nevertheless, may be improved using these ones [7]. More precise techniques and complex criteria require more trained and qualified practitioners.

Thus, dermatoscopy opens up a new dimension on clinical morphology of pigmented skin lesions and enables well-trained physicians to improve their diagnostic accuracy up to $65 \%$ [8]. The use of dermatoscopy both improves clinical diagnostic accuracy and increases the ratio of skin cancers to all lesions biopsied or excised; moreover, this improvement is independent of the type of doctor using dermatoscopy [9]. Also, dermatoscopy in the hands of physicians with experience has higher discriminatory power than nakedeye examination to detect melanoma [10]. Despite all the advantages, dermatoscopy analysis still requires well-trained physician to handle every suspicious skin neoplasm, and thus is not suitable for mass screening applications. Further increase in accuracy of skin lesions analysis may be performed with application of spectroscopic and automatic analysis of acquired data, as digital follow-up examinations and computer-aided diagnosis provide the opportunity to perform precise mass cancer screening without well-trained physicians.

As an alternative to the dermatoscopy a hyperspectral imaging technique may be used. This technique simultaneously allows for imaging of the pathology area and spectral data acquiring. The tissue image allows one to estimate tissue morphology and allows for finding the stage of tumor growth, while the spectral data allows one to make a conclusion about the chemical composition of the tested tissue area. Hyperspectral imaging (HSI) provides information about the intensity of the reflected light at a range of wavelengths at each surface point of the image. The hyperspectral image is a three-dimensional array of $\mathrm{M} \times \mathrm{N} \times \mathrm{K}$ data consisting of a sequence of $\mathrm{K}$ images of $\mathrm{M} \times \mathrm{N}$ size, and each of these images corresponds to an intensity of backscattered signal in the spectral band $\lambda_{\mathrm{K}} \pm \Delta \lambda$ at each point of tested object surface. The applicability of multi- and hyperspectral imaging techniques for the analysis of cancers in visible and NIR ranges has been demonstrated in many studies $[11,12]$. However, accuracy of various cancer pathologies diagnosis with hyperspectral methods implementation for the analysis of backscattering spectra is still remains under consideration.

In this study, the HSI technique was used to demonstrate the possibility of malignant and benign skin tumors differentiation based on the backscattered radiation analysis only in visible spectrum. In contrast to existing methods for diagnosing melanocytic skin tumors that use data on backscattered spectra in the visible and IR regions (see, for example, Ref. [12]), it is proposed to use data from backscattered spectra in a relatively small band of visible spectrum of $520-670$ $\mathrm{nm}$. This approach makes it possible to simplify the system of hyperspectral images recording, which ultimately will reduce the cost of the equipment used in screening surveys. The paper presents data about the analysis of tissues optical density (OD), melanin and hemoglobin content for skin malignant and benign tumors, and application of melanin and hemoglobin content data for skin tumors differentiating.

\section{Materials and Methods}

\subsection{Hyperspectral imaging}

Hyperspectral images of tissues were acquired with HSI camera developed by the Scientific and Technical 
Center of Unique Instrumentation of Russian Academy of Sciences (Moscow) [13]. The setup for hyperspectral images registration is shown in Fig.1. The experimental setup includes the LED source 1, illuminating the tested tissue 2. Backscattered radiation 3 passes through the collimator 4 and enters into the acoustooptic tunable monochromator 5 , controlled by an external computer 8 . The controller board 14 sets the operating mode of high frequency generator 17 and amplifiers 15-16. The generator 17 and the amplifiers $15-16$ creates a high frequency signal supplied to the acoustooptical cells 10 and 12 that generate acoustic waves of a certain frequency. During the passage of an acoustic wave through the acoustooptic cell, optical properties are changed so a filtration of radiation wavelengths occurs, and only radiation at wavelength $\lambda$ reaches the matrix of digital camera 7 . The radiation at wavelengths, that different from $\lambda$, changes direction of its propagation and does not reach the camera. The optical system can display images with spectral resolution up to $2.3 \mathrm{~nm}$ in the range $450-750 \mathrm{~nm}$. The data transfer protocol provides the capability to obtain images with resolution $1360 \times 1024 \mathrm{px}$ (the maximal increasing on scanning area is $7 \times 7 \mathrm{~cm}$ ). Captured hyperspectral images of skin tissues were from $5 \times 5 \mathrm{~cm}$ to $7 \times 7 \mathrm{~cm}$ and contained tumor and healthy tissue area. For methodology optimization, we choose $5 \mathrm{~nm}$ and $501 \times 501 \mathrm{px}$ for scanning step and registration area respectively during the in vivo registration process of the patient's skin hypercube. Thus, each sample is characterized by the hypercube $501 \times 501 \times 61 \mathrm{px}$. Backscattering spectra were stimulated by white LED source located in a distance of $40-50 \mathrm{~cm}$ from the tissue sample. The obtained data has been processed in MATLAB R2014a.

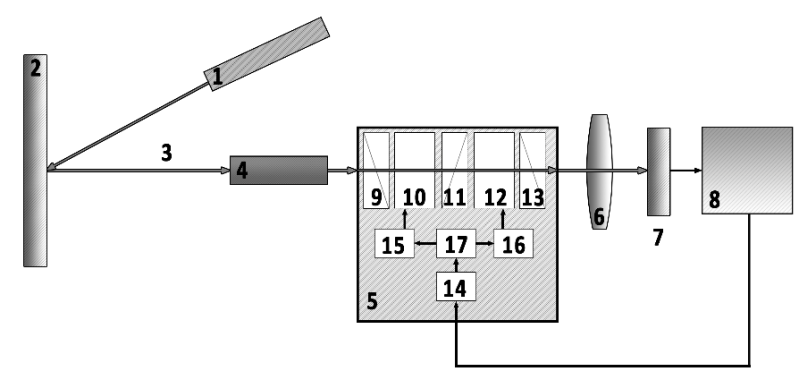

Fig. 1 Scheme of skin hyperspectral images experimental registration: 1 - broadband LED source; 2 - object; 3 - backscattered radiation; 4 - collimator; 5 - tunable monochromator; 6 - lens; 7 - digital camera; 8 - computer; 9, 1113 - polarizers; 10, 12 acoustooptical cells; 14 - controller; 15, 16 - high frequency amplifiers; 17 - high frequency generator.

There were 60 patients with skin cancers (38 female and 21 male, all white, Caucasian, skin phenotype I and II) enrolled in this study. A total of 10 basal cell carcinomas (BCC), 12 malignant melanomas (MM) and 37 benign neoplasms (BN) (including 13 nevi, 4 gemangiomas, 2 inflammatory lipogranulomas, 8 keratosis, 1 Bowen disease, 4 keratopapillomas and 5 different inflammatory diseases) were studied. The detailed distribution of human skin lesions, including information about tumor locations, is provided in Table 1. Every hyperspectral tumor study was accompanied by histological analysis to make a final diagnosis. The protocols of in vivo tissue diagnostics were approved by the ethical committee of Samara State Medical University. The institutional Review Board of Samara National Research University approved the study protocols. This research adhered to the tenets set forth in the Declaration of Helsinki. Informed consent of each subject was obtained.

Typical normalized spectra and tumor images stabilization procedure may be found elsewhere [14, 15]. Generally, to obtain quantitative estimations of various human skin tissues backscattered spectra we used data about OD of skin tissues. The diffuse backscattered reflectance from skin in spatial point $(x$, y) may be characterized by $\mathrm{OD}$ on the selected wavelength $\lambda$ as:

$$
O D(x, y, \lambda)=\lg \frac{I_{o}(x, y, \lambda)}{I(x, y, \lambda)}
$$

where $I_{0}(x, y, \lambda)$ is backscatter intensity from the background of light source, $I(x, y, \lambda)$ is backscattered intensity from the tissue sample. While the exact determination of tumor type may be performed with OD analysis based on above spectral bands:

$$
O D_{i}(x, y)=\frac{1}{\lambda_{2}-\lambda_{1}} \int_{\lambda_{1}}^{\lambda_{2}} O D(x, y, \lambda) d \lambda
$$

where hemoglobin-related optical density $O D_{H}$ defined for $\left(\lambda_{1}=530 \mathrm{~nm}, \lambda_{2}=570 \mathrm{~nm}\right)$ and melanin-related $O D_{M}$ for $\left(\lambda_{1}=600 \mathrm{~nm}, \lambda_{2}=670 \mathrm{~nm}\right)$. Further, we calculated mean $O D_{H}$ and $O D_{M}$ values for all studied tumors and healthy skin. Allocation of tumor area was performed on the basis of approach presented in our previous studies [15]. Healthy skin region was chosen near the tumor area with $1.5-2 \mathrm{~cm}$ indentation from the tumor and included approximately $1 \times 1 \mathrm{~cm}$ area of healthy skin. Example of hyperspectral image with tumor and healthy skin areas allocation as well as distribution of $O D_{M}$ coefficient is presented in Fig. 2.

Discrimination of tumors was performed based on two approaches. First approach took into account only raw values of $O D_{H}$ and $O D_{M}$ coefficients, while second approach used normalized $O D^{N}{ }_{H}$ and $O D^{N}{ }_{M}$ coefficients. Normalization was performed as:

$$
O D_{i}^{N}=\frac{O D_{i}^{T}}{O D_{i}^{H}}
$$

where $O D^{T}{ }_{i}$ and $O D^{H}{ }_{i}$ are $O D_{i}$ coefficients calculated for one patient for tumor (index ${ }^{T}$ ) and healthy skin (index ${ }^{H}$ ) areas respectively.

$O D_{i}$ coefficients were used for tissue classification by discriminant analysis (DA). DA can separate two or more classes based on different statistical parameters of 
Table 1 Summary of patients with skin tumors.

\begin{tabular}{|c|c|c|c|c|c|c|c|}
\hline \multirow{2}{*}{ Lesion } & \multirow{2}{*}{ Age } & \multicolumn{2}{|c|}{ Gender } & \multicolumn{4}{|c|}{ Location } \\
\hline & & Male & Female & chest/stomach & neck/head & limb & back \\
\hline MM & $39-77$ & 4 & 8 & 2 & - & 4 & 6 \\
\hline $\mathrm{BCC}$ & $36-82$ & 6 & 4 & 1 & 5 & 1 & 4 \\
\hline $\mathrm{BN}$ & $21-84$ & 11 & 26 & 5 & 6 & 9 & 13 \\
\hline
\end{tabular}

Gaussian distributions. The efficiency of the proposed approach is characterized by sensitivity and specificity and ability to select defined classes in different areas of phase plane. The analysis of skin tissues data allocation was performed using quadratic DA classifiers [16].

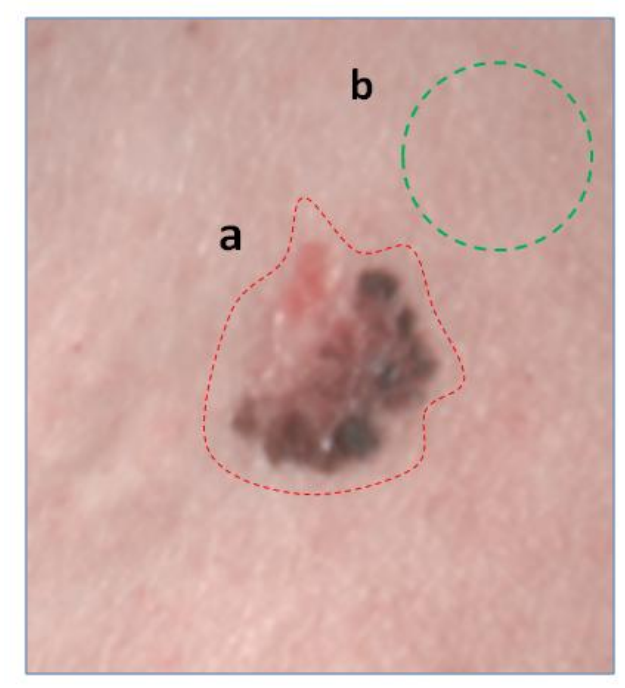

Fig. 2 Hyperspectral image of in vivo skin sample containing MM (a) and healthy skin (b) regions $(5 \times 5$ $\mathrm{cm})$

\subsection{Diffuse-reflectance spectra measurement}

For reflectance measurements a halogen lamp with broad-band output spectrum $(370-1050 \mathrm{~nm})$ was applied. Optical fiber probe R400-7-VIS-NIR (400um Reflection Probe, VIS/NIR, 2m (Ocean Optics Inc., Dunedin, FL, USA) was used to deliver the light from laser and lamp and to collect the diffuse reflectance signals. The spectra were recorded and stored using a fiber-optic microspectrometer (USB4000-VIS-NIR, Ocean Optics, Dunedin, FL, USA). A personal computer was used to control the system and to store and display the data using the specialized microspectrometer software SpectraSuite ("Ocean Optics", Inc., Dunedin, FL, USA).

For reflectance measurement mode initial calibration was carried out before every measurement. The portion of the light reflected from a sample is expressed as a percentage $R[\%]$ relative to a standard reflectance (Spectralon $($ )). The reflectance $R[\%]$ is calculated by the following equation:

$$
R_{\lambda}=\frac{S_{\lambda}-D_{\lambda}}{S R_{\lambda}-D_{\lambda}} \times 100 \%,
$$

where $R_{\lambda}$ is the reflectance [\%], $S_{\lambda}$ is the light intensity reflected by the sample at each wavelength, $D_{\lambda}$ the dark intensity at each wavelength (correction signal for detector dark current), $S R_{\lambda}$ the standard reference reflectance intensity at each wavelength.

Spectroscopic measurements of normal skin and lesion areas were carried out after 5-10 minutes of rest for each patient at room temperature (23 to $\left.25{ }^{\circ} \mathrm{C}\right)$. Several spectra were measured from each suspicious area and averaged to reduce the influence of inhomogeneity of the lesions. It was recorded and averaged five to seven spectra from every lesion, depending on its size, and three to five spectra from surrounding normal skin. These averaged spectra from the health skin area were used like an indicator of the spectral changes in the pathological areas. The spectra were smoothed using the Savitzky-Golay algorithm in order to reduce the instrumental noise of the spectrometric system. A constant distance of $2 \mathrm{~mm}$ between the end tip of the optical fibers and the skin surface were applied, using a mechanical stand to avoid any influence of displacement from the normal position on the intensity level. All spectra were obtained at normal incidence - at the $90^{\circ}$ angle between optical fibers end tip and skin surface. This geometry was carefully reproduced in all measurements to minimize diffuse reflectance intensity changes related to distance, and reflectance spectral shape changes related to the angle uncertainties during measurements.

Before every spectroscopic measurement a clinical observation and dermatoscopic evaluation of the lesion under interest was made. After these initial medical and spectroscopic measurements a histological samples were obtained from every lesion. The results from histological examination were used as a "gold standard" in comparison of all data obtained.

In this study we had included diffuse reflectance results from 102 patients, distributed as follow: 39 benign nevi, 35 -dysplastic nevi, 28 - pigmented malignant melanoma (nodular and lentigo MM).

\section{Results and Discussion}

In the visible region of the spectrum (400-800 nm), the main chromophores of biological tissues are hemoglobin and melanin, since proteins absorb radiation in the shorter-wave and longer-wave regions, 
water in the longer-wave region, and fats have a local absorption maximum near $750 \mathrm{~nm}[17,18]$. The presence of such a transparency window of biological tissues allows for studying the internal structure of a thin surface layer of tissue with up to several millimeters depth [19]. In this case, the dependence of the extinction coefficient of melanin has a descending shape in the $450-750 \mathrm{~nm}$ region, while hemoglobin extinction coefficient in the same spectral region has two local maxima near the $530 \mathrm{~nm}$ and $570 \mathrm{~nm}$ regions. Moreover, upon transition to a longer wavelength region (600-800 nm), the value of the extinction coefficient of hemoglobin is much smaller and becomes several orders of magnitude lower than extinction melanin in the same region. Thus, to estimate hemoglobin and melanin content in the skin we calculated $O D_{H}$ coefficient in 530 - $570 \mathrm{~nm}$ area and $O D_{M}$ in $600-670 \mathrm{~nm}$ area.

In diffuse-reflectance spectroscopy mode the most significant differences are related to the influence of skin pigments - melanin and hemoglobin from the blood. They are responsible for the shape of the reflectance spectra observed in the region of 350-1000 nm. On Fig. 3 are presented averaged, compared by pathology type lesions vs. normal skin reflectance. Malignant melanoma (MM) lesions (28 patients) had the lowest reflectance intensity in the whole spectral range in VIS-NIR region. The dysplastic nevi reflectance is similar by intensity levels and could not be easily separated by values from MM, but the spectral shape differences observed, related to a reduced slope values of the reflectance curve in the region of 600-900 $\mathrm{nm}$ is typical for the non-melanoma pigmented lesions, while for a normal skin it is expressively negative by value and strongly positive for all pigmented melanoma lesions detected.

Thus, differentiation of pathologies from normal tissues and distinguishing one type of tumor from the other is possible with backscattered spectrum analysis in the visible region, since it contains data on hemoglobin and melanin content in skin tissues. In current study for the differentiation of skin tissues we used values of OD for melanin and hemoglobin content. Fig. 4 shows distributions of $O D_{H}$ and $O D_{M}$ coefficients for $\mathrm{BN}$ and malignant skin tissues. One may see that MM and BCC have almost the same values of $O D_{H}$. This fact proves

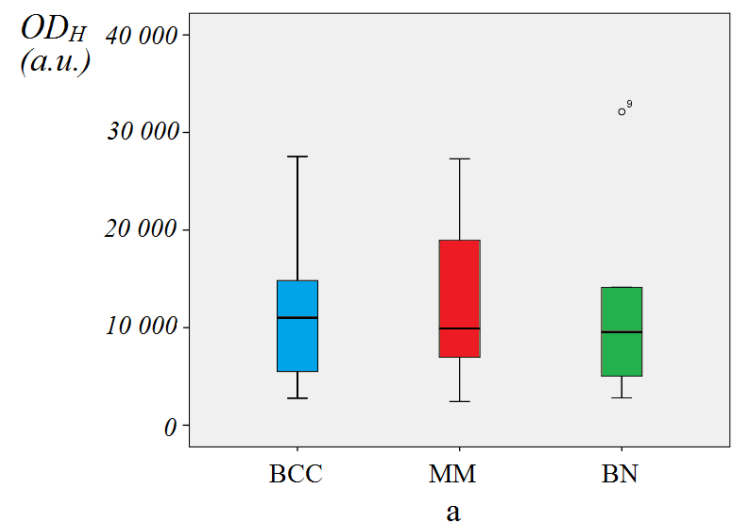

our assumption about vast capillary network in malignant tumors in comparison to $\mathrm{BN}$, as $\mathrm{BN}$ formations contain less blood according to Fig. 4 (a) data. For melanin content (Fig. 4 (b)) we may note a vast dispersion for $\mathrm{BCC}$ data, but this fact may be caused by rather small number of studied BCCs. Also, it is interesting to note that medians for $O D_{H}$ and $O D_{M}$ distributions for all three studied skin classes (MM, $\mathrm{BCC}$ and $\mathrm{BN}$ ) have almost identical values.

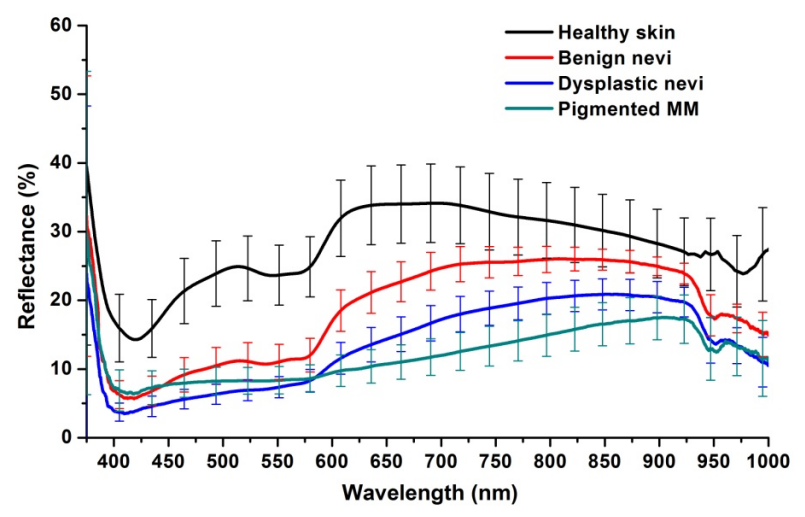

Fig. 3 Diffuse reflectance spectra of melanin-pigmented lesions vs. healthy skin tissue reflectance. Spectra are averaged by the set of lesions of different pathologies investigated and the standard deviation is presented as error bars.

In general, application of $O D_{H}$ and $O D_{M}$ coefficients shows accuracy of skin tissues classification on $55-60$ $\%$ level. Increase of skin tissues classification accuracy is possible with joint implementation of two criteria. This approach may be implemented with phase plane analysis. In separation of phase plane classes axes of the plane are criterions of tissues classification, and every tested sample is a point on the phase plane with coordinates corresponding to the criterion values for this tissue sample [20]. Phase plane analysis was performed for $O D_{H}$ and $O D_{M}$ coefficients pair. Example of phase plane analysis is shown in Fig. 5. Separation of BCC and $\mathrm{MM}$ was performed with quadratic DA, axes of phase plane in Fig. 5 are $O D_{H}$ and $O D_{M}$.

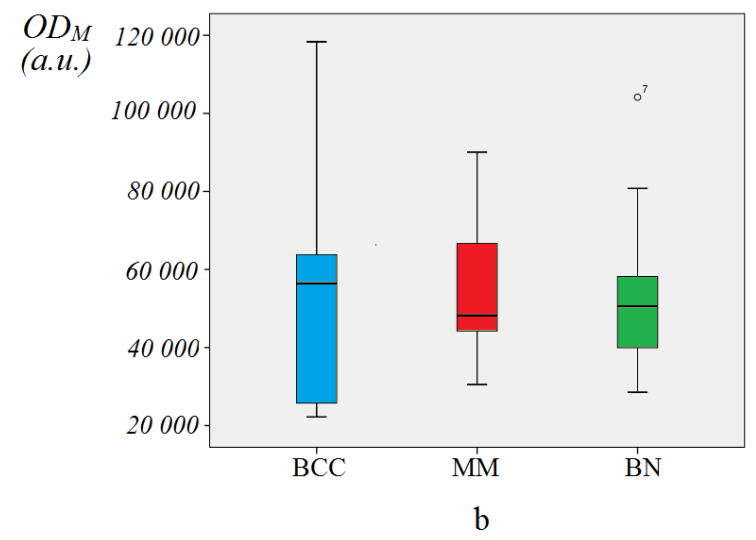

Fig. 4 Whisker and box plots for $O D_{H}(\mathrm{a})$ and $O D_{M}(\mathrm{~b})$ distribution of skin tissues. 


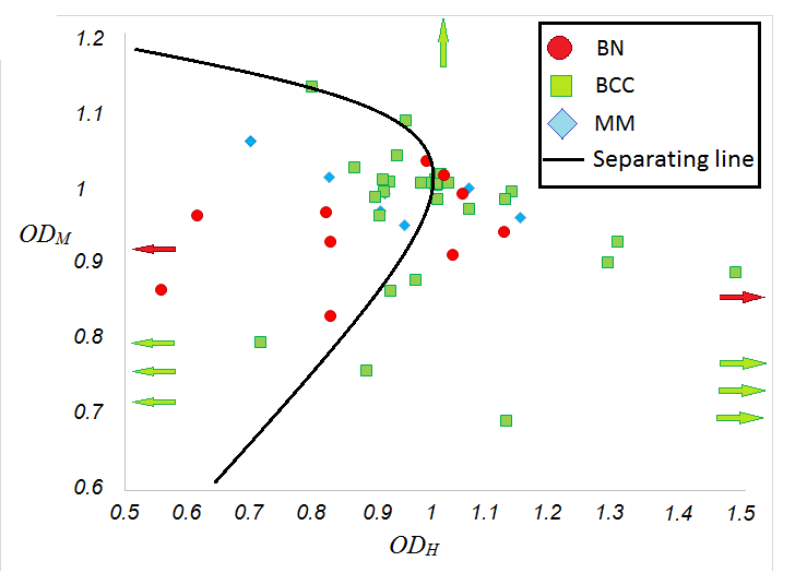

a

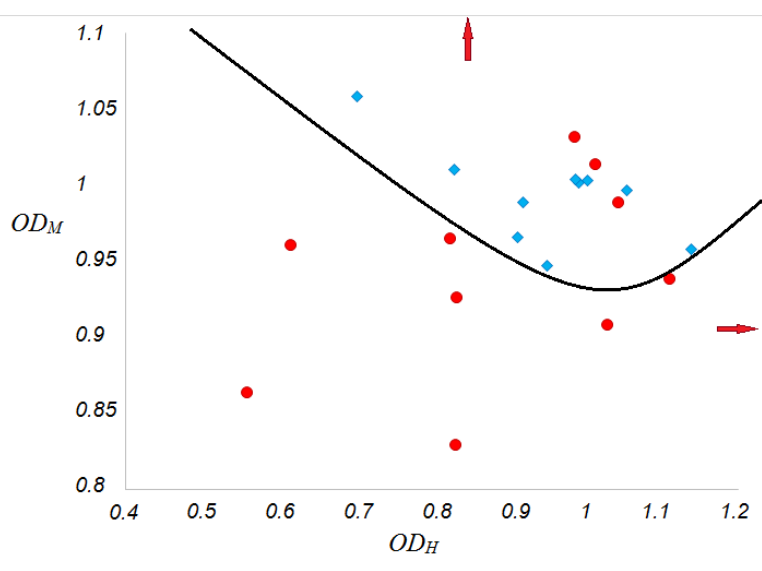

b

Fig. 5 Skin neoplasms separation on phase plane with $O D_{H}$ and $O D_{M}$ coefficients and quadratic DA: malignant tissues vs BN (a) and MM vs BCC (b).
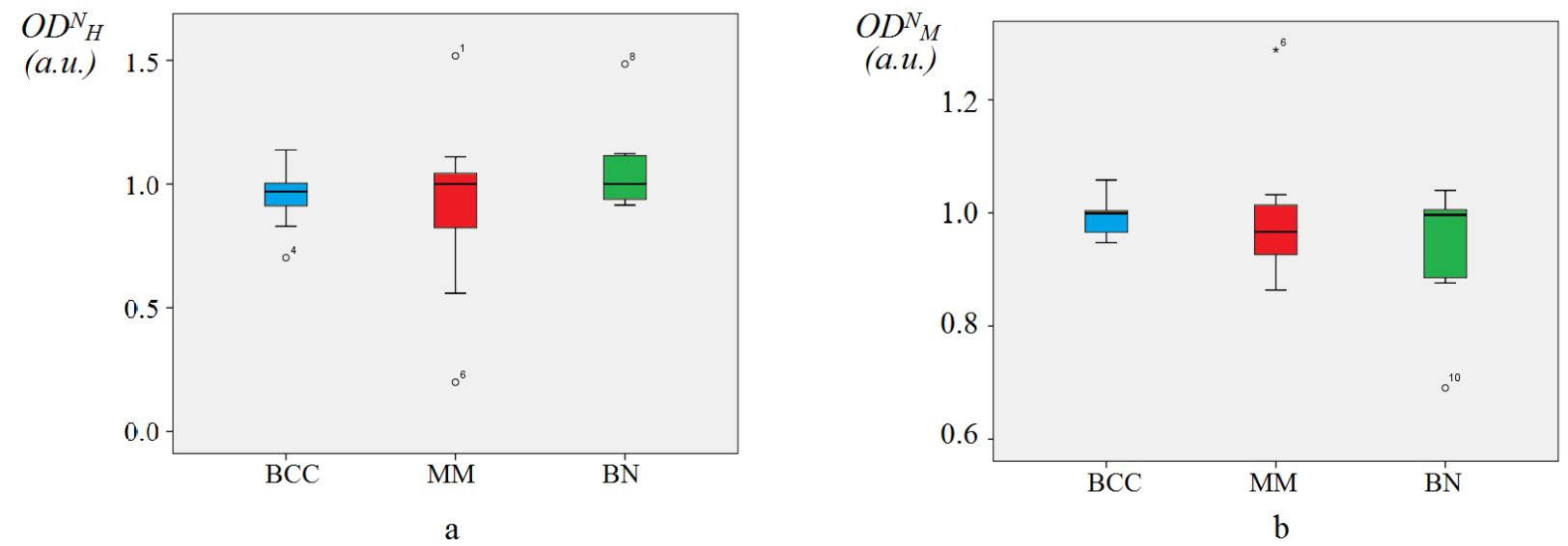

Fig. 6 Whisker and box plots for $O D_{H}^{N}$ (a) and $O D_{M}^{N}$ (b) distribution of skin tissues.

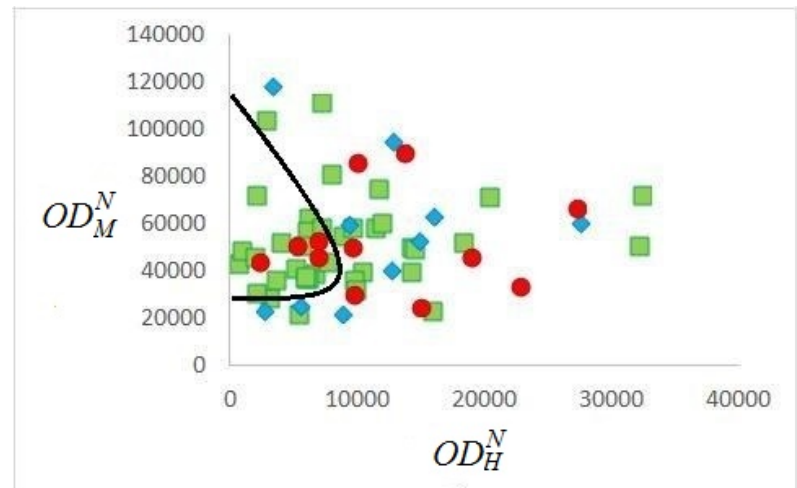

a

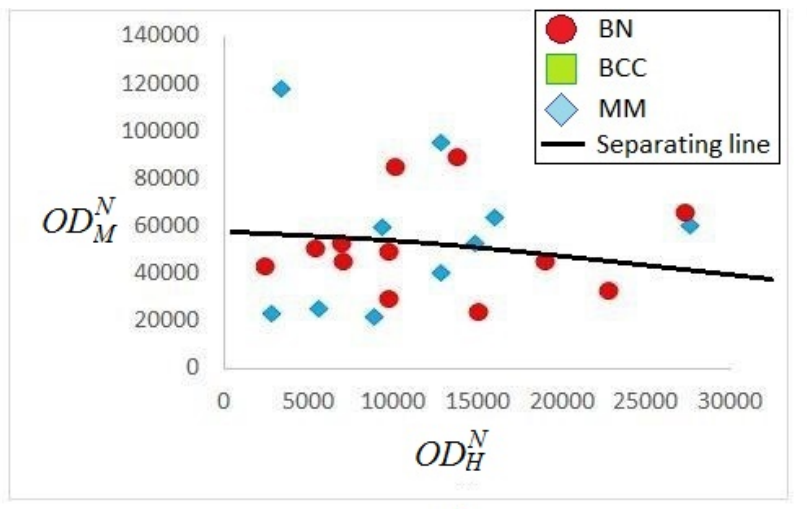

b

Fig. 7 Skin neoplasms separation on phase plane with $O D^{N}{ }_{H}$ and $O D^{N}{ }_{M}$ coefficients and quadratic DA: malignant tissues vs BN (a) and MM vs BCC (b).

Both classifications presented in Fig. 5 (malignant vs $\mathrm{BN}$ and $\mathrm{MM}$ vs $\mathrm{BCC}$ ) provides $68 \%$ accuracy. Here sensitivity and specificity for malignant vs BN classification are $82 \%$ and $54 \%$ respectively; and sensitivity and specificity for MM vs BCC classification are $75 \%$ and $60 \%$ respectively. Rather low accuracy of about $70 \%$ in skin tissues classification in comparison with other optical techniques application for skin pathologies analysis [21-23] may be caused by high dispersion in melanin and hemoglobin content in skin 
for different persons. A number of studies demonstrates a clear contrast between skin phototypes, skin sites, and wavelengths for main skin chromophores concentrations [24]. Thus, we may take into account initial variations of skin properties and count these variations in $O D_{H}$ and $O D_{M}$ coefficients calculation.

In order to account initial skin chromophores concentrations we may normalize $O D_{H}$ and $O D_{M}$ coefficients for tested tumors on the values of the same coefficients for normal skin of the same patient. Details of normalized $O D^{N}{ }_{H}$ and $O D^{N}{ }_{M}$ coefficients calculation are presented in section 2.3. Fig. 6 demonstrates whisker and box plots for $O D^{N}{ }_{H}$ and $O D^{N}{ }_{M}$ distributions for skin tissues. Comparison of Fig. 4 and Fig. 6 shows that normalization of $O D_{H}$ and $O D_{M}$ on the values of normal skin helps to slightly improve the separation of skin tissues classes based on melanin and hemoglobin content calculation.

Application of $O D^{N}{ }_{H}$ and $O D^{N}{ }_{M}$ coefficients for skin tissues separation helps to achieve about $60-70 \%$ accuracy, that is $5-10 \%$ higher than for the $O D_{H}$ and $O D_{M}$ coefficients implementation. Analysis of phase planes for malignant and $\mathrm{BN}$ tissues separation and $\mathrm{MM}$ and BCC separation is presented in Fig. 7. Here separation of malignant and $\mathrm{BN}$ tissues is possible with sensitivity and specificity of $64 \%$ and $73 \%$ correspondingly and total accuracy of $69 \%$. This accuracy is almost the same as in the case of $O D_{H}$ and $O D_{M}$ coefficients application. However, separation of $\mathrm{MM}$ and $\mathrm{BCC}$ is possible with sensitivity and specificity of $75 \%$ and $100 \%$ correspondingly and total accuracy of $88 \%$. Additionally, it is interesting to note, that separation of $\mathrm{MM}$ and $\mathrm{BN}$ is possible with $68 \%$ accuracy for $O D_{H}$ and $O D_{M}$ coefficients, and with $74 \%$ accuracy for $O D^{N}{ }_{H}$ and $O D^{N}{ }_{M}$.

Comparing the proposed method with the previously developed methods of MM instrumental detection [11, $22,25,26]$ one may say that the developed method can compete not only with other hyperspectral methods of analysis of biological tissues, but also with such types of optical analysis of biological tissues as an autofluorescence analysis and Raman spectroscopy. Despite slightly less values of total accuracy for skin tissues classification in comparison to Raman spectroscopy study [21, 23], hyperspectral imaging provides an ability to scan large tissue areas and detect suspicious formations. Raman spectroscopy today commonly used to measure tissue properties only in a single point [27-29]. The proposed hyperspectral imaging setup allows for achieving almost the same results of $\mathrm{MM}$ and $\mathrm{BN}$ separation when compared with hyperspectral techniques using data both from visible and NIR spectra [12]. Although, to make a reliable comparison of the results of the proposed method with the data of other studies, more wide studies on the registration and analysis of hyperspectral images of skin tumors are required.

\section{Conclusion}

The developed method of human skin tumors differentiation may become a simple and reliable approach for mass screening applications, as it allows for quickly detection and classification of malignant tumors with relatively inexpensive equipment. Registration of tissue hyperspectral image is possible within 15-20 seconds, so the proposed technique may be used directly in clinics. In addition, in the proposed approach, only the white LED light illuminator is used as a radiation source. All of this makes the system used in the study a reliable, quick and inexpensive device of skin mass screening applications.

Comparison of the achieved results with the existing hyperspectral methods of skin pathologies detection [12] allows us to say that the developed approach makes it possible to confidently detect malignant tumors and uses for this purpose only the spectral response of the skin in visible spectrum. Comparison of similar approaches of skin tumors diagnostics using information about backscattered radiation in the visible spectrum [25] also supports the proposed experimental technique. Dicker et al [25] applied hyperspectral imaging in the visible spectra only for ex vivo diagnostics of pigmented skin tumors, while the proposed method successfully work with real patients in clinics. In addition, the proposed method can become a part of a more complex system of tissues optical monitoring. This may be done by combining the results of the study with data obtained by other optical methods [27, 30].

Further development of the proposed system is possible in several directions. The first is to conduct more wide in vivo studies both with samples of skin tumors and with other human tissues samples to establish the exact diagnostic capabilities of the proposed technique. The second direction is the creation of an automatic algorithm for the detection of tumors in hyperspectral images, which would allow for determining the tumor presence and analysis of tumor type based on the data of backscattered spectra. Thus, the proposed approach to mass screening of skin tumors may significantly increase the accuracy of oncological pathologies diagnosis during the "first-hand" examinations in clinics.

\section{Disclosures}

All authors declare no conflict of interests for this paper and have no financial interest in the materials used in the chapter.

\section{Acknowledgements}

This research was supported by the Ministry of Education and Science of the Russian Federation. 